\title{
RT-qPCR versus Digital PCR: How Do They Impact Differently on Clinical Management of Chronic Myeloid Leukemia Patients?
}

\author{
Camilla Zanaglio ${ }^{a, b}$ Simona Bernardi ${ }^{a, b} \quad$ Lisa Gandolfi $^{a} \quad$ Mirko Farina $^{a}$ \\ Federica $\operatorname{Re}^{a}{ }^{b}$ Nicola Polverellia Tatiana Zollner ${ }^{a}$ Alessandro Turra ${ }^{a}$ \\ Enrico Morello ${ }^{a}$ Michele Malagola ${ }^{a}$ Domenico Russo ${ }^{a}$ \\ ${ }^{a}$ Chair of Hematology, Unit of Blood Diseases and Stem Cell Transplantation, Department \\ of Clinical and Experimental Sciences, University of Brescia, ASST Spedali Civili di Brescia, \\ Brescia, Italy; ${ }^{b}$ CREA Laboratory (Centro di Ricerca Emato-Oncologica AIL), ASST Spedali \\ Civili di Brescia, Brescia, Italy
}

\author{
Keywords \\ Chronic myeloid leukemia - Digital PCR - Minimal residual disease monitoring $\cdot R T-q P C R$. \\ BCR-ABL1
}

\begin{abstract}
Real-time quantitative PCR (RT-qPCR) is the gold standard to quantify the BCR-ABL1 transcript for molecular response monitoring in chronic myeloid leukemia $(\mathrm{CML})$ patients, and it plays a pivotal role in clinical decision-making process, even if it presents technical limits. Increasing data suggest that digital PCR ( $d P C R)$ is more accurate and reliable than RT-qPCR in CML minimal residual disease monitoring and in patients' selection for treatment discontinuation. But what about the identification of treatment discontinuation failures? We present the case of a CML patient enrolled both in a study aiming to comparatively assess molecular response by RT-qPCR and dPCR and in the progressive arm of the OPTkIMA trial. This is a phase III trial including CML patients randomized to receive a fixed versus a progressive intermittent tyrosine kinase inhibitor regimen. At 24 months, because of two consecutive detections of $\mathrm{MR}^{2.0}$ by RT-qPCR, the patient resumed daily treatment. Conversely, dPCR revealed a stability of molecular response and even a slight decreasing of transcript over time. An additional specimen was sampled one month after the first $M^{2.0}$ detection because of clinical decision: RTqPCR resulted MR ${ }^{3.0}$ and $d P C R$ confirmed the transcript's stability. Nowadays, the resumption of therapy is RT-qPCR-driven despite its limits in detection and robustness. In this case, according to $\mathrm{dPCR}$, the patient could have continued intermittent treatment and the stability of response was then confirmed by RT-qPCR. So, dPCR could be able to better identify peculiar clinical response to therapy.




\section{Introduction}

Philadelphia-positive chronic myeloid leukemia (CML) is marked by chromosome translocation $\mathrm{t}(9 ; 22)(\mathrm{q} 22 ; \mathrm{q} 11)$ that leads to the gene $B C R-A B L 1$. This gene, encoding for a deregulated tyrosine kinase protein (p210), drives the leukemic transformation of hematopoietic stem cells and induces the progression of the disease from the early chronic phase to the fatal blastic phase [1-6]. Tyrosin kinase inhibitors (TKIs) were introduced in the 2000s, and they changed the fate of CML patients by avoiding the disease's blastic transformation and significantly improving survival.

More than $80 \%$ of all patients treated with TKIs have now a life expectancy comparable to that of the general population [2]. In this context, the scientific community is facing new objectives: achieving a faster major molecular response (MMR) to prevent progression to blastic phase, obtaining a stable deep molecular response (DMR) to attempt treatment discontinuation, and improving quality of life $[1,3,7]$.

According to the latest European and American guidelines, MMR or $\mathrm{MR}^{3.0}$ is defined as BCR-ABL1/ABL1 international scale (IS) $\leq 0.1 \%$ by real-time quantitative PCR (RT-qPCR), and $\operatorname{DMR}\left(\geq \mathrm{MR}^{4.0}\right)$ is defined as BCR-ABL1/ABL1 IS $\leq 0.01 \%$ [8]. RT-qPCR became the gold standard to monitor minimal residual disease (MRD), and it plays an essential role in the management of CML patients, as the achievement of such disease response is a surrogate for survival and may guide the decision for treatment modification [8, 9]. However, RT-qPCR has some intrinsic limits, related to its sensitivity and accuracy, and these limitations do not facilitate the evaluation of stable DMR and the selection of the best candidates for TKI discontinuation $[10,11]$.

In the recent years, digital PCR (dPCR) has appeared as a more sensitive and accurate tool for the detection of MRD in CML. Although it is not yet routinely applied, increasing data suggest that dPCR may be more than an alternative approach to RT-qPCR due to a greater capacity to measure low concentrations of the two transcript variants (b3a2 and b2a2), to improve the recognition of stable DMR, and, possibly, the selection of patients for treatment discontinuation and the prediction for treatment-free remission (TFR) [12-17].

\section{Case Report}

We present the case of an 80-year-old female, diagnosed in April 2015 with chronic phase CML (b3a2) with intermediate Sokal risk. Imatinib (IM) treatment was started at $300 \mathrm{mg}$ daily. After six months, she achieved DMR $\left(\mathrm{MR}^{4.0}\right)$ with complete cytogenetic response. After 12 months of treatment, BCR-ABL1 transcript resulted undetectable (MR ${ }^{4.5 \mathrm{U}}$ ) by RT-qPCR. The IM dose was decreased from $300 \mathrm{mg} /$ day to alternate doses of 300-200 mg/day because of grade 2 fluid retention and grade $1 / 2$ dyspepsia.

In February 2016, our patient gave her written informed consent to be enrolled in the study aiming to comparatively assess BCR-ABL1 transcript levels by RT-qPCR and APCR (NP-DPCR-CML_1603 - Ethical Committee approval) [12,16]. In January 2018, the patient full filled the inclusion criteria to be enrolled in the OPTkIMA trial (ClinicalTrials.gov: NCT02326311). This is an ongoing prospective multicentric phase III randomized trial including CML patients $>60$ years with stable ( $\geq 2$ years) $\mathrm{MR}^{3.0}$ or $\mathrm{MR}^{4.0}$ molecular response, randomized 1:1 to receive a fixed intermittent TKI regimen (one month $\mathrm{ON}$ and one month OFF), on the basis of interim study results $[18,19]$, versus a progressive intermittent TKI regimen (one month $\mathrm{ON}$ and one month OFF for the first year; one month $\mathrm{ON}$ and two months OFF for the second year; one month $\mathrm{ON}$ and three months OFF from the third year onwards). Conventional MRD monitoring is scheduled every 3 months, and in case of confirmed MMR loss at two consecutive time points, patients are planned to resume TKIs daily.

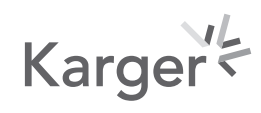




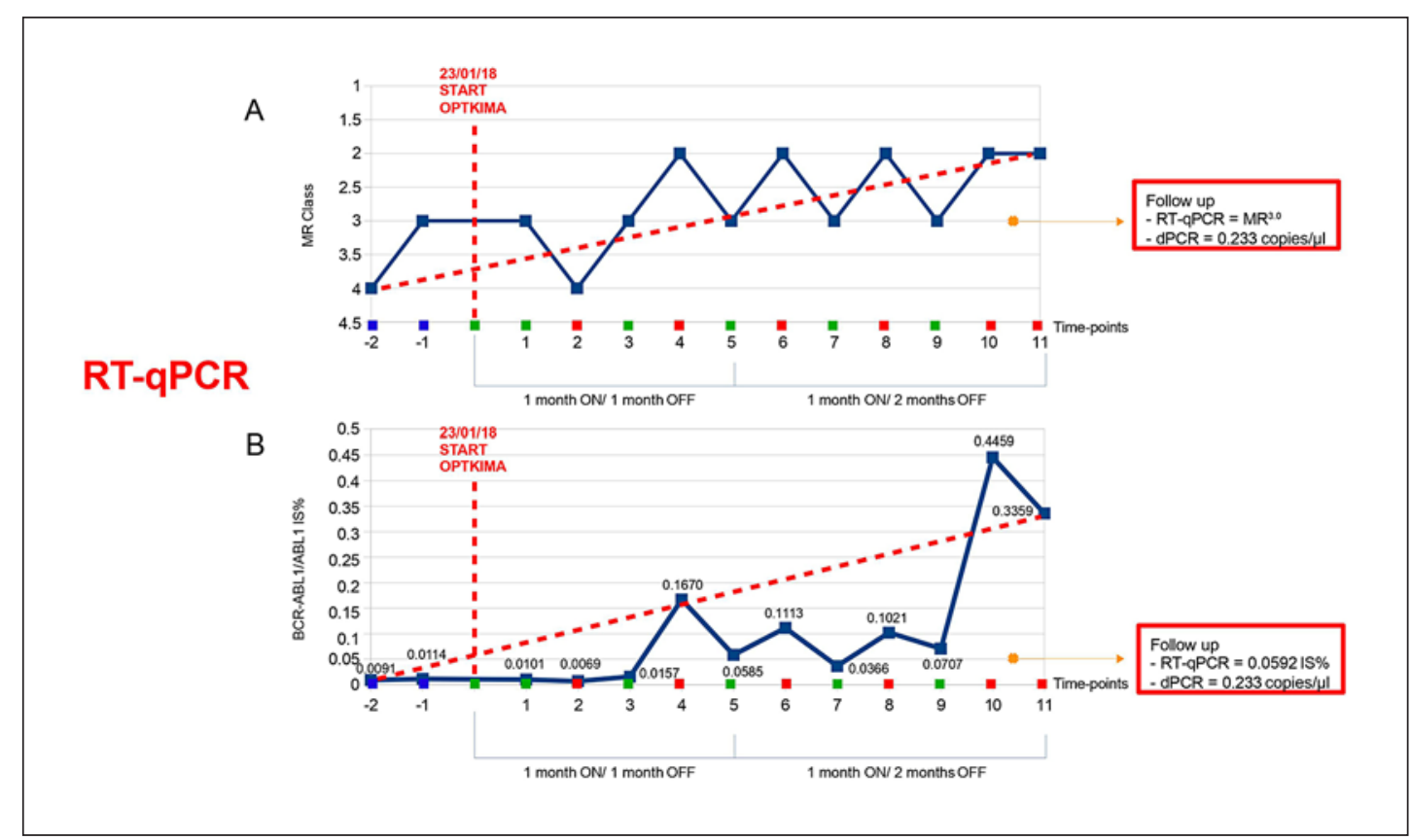

Fig. 1. MRD monitoring by RT-qPCR. The figure reports the MRD monitoring performed by RT-qPCR. The results of the RT-qPCR monitoring are expressed as MR classes (A) and as IS values in percentage (B). Blue squares represent time points before the enrollment in the OPTkIMA protocol. Green squares represent time points in the months of imatinib treatment. Red squares represent time points in the months without imatinib treatment. The red dashed lines represent the RT-qPCR monitoring trends.

At enrollment, the patient was in the $\mathrm{MR}^{4.0}$ class (IS: $0.0091 \%$ ), and she was randomized to the progressive arm. At each time point scheduled for MRD monitoring (every 3 months), $10 \mathrm{~mL}$ peripheral blood were sampled and used for RT-qPCR and APCR analysis [8, 20]. BCR-ABL1 transcript was quantified by conventional RT-qPCR at the GIMEMA-Labnet reference laboratory of ASST Spedali Civili of Brescia, according to ELN guidelines [3, 8, 21, $22]$. The dPCR analysis was performed as previously described $[12,16]$ at the same reference laboratory. All samples were analyzed twice by different operators, and the final results were expressed as means of the number of $B C R-A B L 1$ copies/ $\mu \mathrm{L}$ of reaction of the replicates $[12,16]$.

The results of the MRD monitoring by RT-qPCR and dPCR are reported in Figures 1 and 2 , respectively. The transcript determinations by RT-qPCR were expressed as MR class (Fig. 1A) and as IS value in percentage (Fig. 1B), while the transcript determinations by dPCR were expressed as number of $B C R-A B L 1$ copies/ $\mu \mathrm{L}$ (Fig. 2). In Figure 2, the dotted line indicates the threshold of $0.468 \mathrm{BCR}-\mathrm{ABL} 1$ copies/ $\mu \mathrm{L}$, previously described as able to identify patients presenting stable DMR $[12,16]$.

The RT-qPCR graph shows that, over the course of 24 months, the MR class moved from $\mathrm{MR}^{4.0}$ to $\mathrm{MR}^{2.0}$ (Fig. 1A) and that the IS value progressively increased from 0.0091 to $0.3359 \%$ (Fig. 1B). In both cases, the worsening of the MR class and of the IS value did not occur linearly but rather in a swinging way. Moreover, in the graph, it may be well appreciated how minimal oscillations of IS value led to a clear jump in the MR class. Interestingly, after six months, $\mathrm{MR}^{3.0}$ class was detected during the months $\mathrm{ON}$ of the intermittent therapy and $\mathrm{MR}^{2.0}$ class during the months OFF of the intermittent therapy, until 24 months when, because of two consecutive $\mathrm{MR}^{2.0}$ detections, the patient resumed daily treatment. The dPCR graph clearly shows a stability of transcript levels over time. All but one dPCR value (at 18 months) were below the 


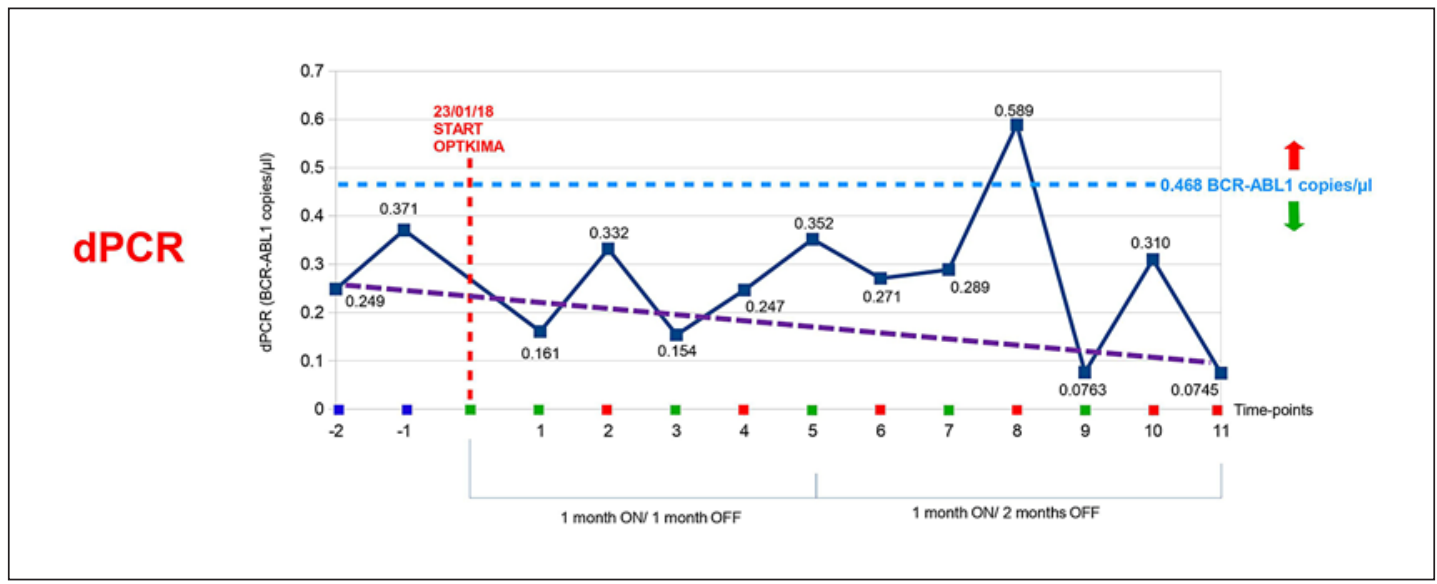

Fig. 2. MRD monitoring by $\mathrm{dPCR}$. The figure reports the MRD monitoring performed by dPCR. the results of the $\mathrm{APCR}$ monitoring are expressed as BCR-ABL1 copies/ $\mu \mathrm{L}$. Blue squares represent time points before the enrollment in the OPTkIMA protocol. Green squares represent time points in the months of imatinib treatment. Red squares represent time points in the months without imatinib treatment. The dark blue dashed line represents the $\mathrm{dPCR}$ monitoring trends. The light blue dashed line represents the threshold of 0.468 BCR-ABL1 copies $/ \mu \mathrm{L}$.

threshold of 0.468 BCR-ABL1 copies/ $\mu \mathrm{L}$, and for this reason the observed oscillations were considered irrelevant (Fig. 2).

On January 8, 2020, our patient resumed her previous daily treatment as required by the OPTkIMA protocol due to MMR loss/MR ${ }^{3.0}$ by RT-qPCR at two consecutive time points. Following clinical decision, an additional specimen was sampled one month after the first $\mathrm{MR}^{2.0}$ detection and was comparatively evaluated by RT-qPCR and dPCR. The result of conventional RT-qPCR was $\mathrm{MR}^{3.0}$, and the dPCR value resulted in $<0.468$ copies $/ \mu \mathrm{L}$ ( 0.233 copies/ $\mu \mathrm{L})$. Furthermore, two additional specimens were sampled one and three months after resumption of daily treatment, they were comparatively evaluated by RT-qPCR and APCR. $\mathrm{MR}^{3.0}$ was detected by RT-qPCR at both evaluations, while dPCR measured values $<0.468$ copies/ $\mu \mathrm{L}$ ( 0.234 and 0.147 copies/ $\mu \mathrm{L}$; data not shown in Figs. 1 and 2).

\section{Discussion}

The study reports on a comparative RT-qPCR/dPCR monitoring of BCR-ABL1 transcript levels in a CML patient enrolled in the OPTkIMA study, an ongoing phase III randomized trial based on fixed or progressive intermittent TKI regimen in elderly CML patients with stable ( $\geq 2$ years) $\mathrm{MR}^{3.0}$ or $\mathrm{MR}^{4.0}$ molecular response. It is known that the gold standard method for evaluating and monitoring the molecular response in CML patients treated with TKIs is RT-qPCR, and also in this trial, the plan of treatment has been designed according to the level of molecular response as detected by RT-qPCR. Thus, conventional monitoring of MRD by RT-qPCR was scheduled every 3 months, and in case of confirmed MMR/MR ${ }^{3.0}$ loss at two consecutive time points, patients are planned to resume TKIs daily.

According to the study design, our patient was enrolled in the progressive arm when in $\mathrm{MR}^{4.0}$ (IS $0.0091 \%$ ). She was treated intermittently one month ON/one month OFF for the first year, and one month ON/two months OFF for the second year. At 24 months, she experienced confirmed $\mathrm{MR}^{3.0}$ loss and had to resume IM daily. Monitoring MRD by RT-qPCR and 
dPCR comparatively, we observed two divergent profiles of molecular response. On one hand, the RT-qPCR profile documented an increasing BCR-ABL transcript, on the other hand, dPCR showed a decrease.

Indeed, by RT-qPCR, the MR class moved from $\mathrm{MR}^{4.0}$ to $\mathrm{MR}^{2.0}$ (Fig. 1A) and the IS value progressively increased from 0.0091 to $0.3359 \%$ (Fig. 1B), over the course of 24 months. On the contrary, by dPCR, the measurements documented not only a stability of transcript levels over time, but even a slight progressive reduction in the number of BCR-ABL1 copies/ $\mu \mathrm{L}$ from 0.249 to 0.0745 . Anyhow, all but one dPCR values (at 18 months) resulted below the threshold of 0.468 BCR-ABL1 copies/ $\mu \mathrm{L}$.

Based on this laboratory trend, the burning question is: where is the truth? For many years, we have been working on testing the sensitivity and reliability of dPCR for the detection and monitoring of MRD in CML. We demonstrated that the levels of BCR-ABL1 as measured by $\mathrm{dPCR}$ are highly heterogenous within each MR class, that levels of transcript can be measured in cases "undetectable" by RT-qPCR, that the threshold $<0.468$ BCR-ABL1 copies/ $\mu \mathrm{L}$ is able to better identify patients with stable DMR, and that values below this cutoff predict TFR. Translating our previous experience concerning the use of dPCR into clinical practice, this patient would be considered as a stable deep responder and candidate to continue the de-escalation of treatment according to protocol.

It has already been reported and discussed in the literature that the higher sensitivity and accuracy of dPCR could allow the inclusion of patients into trials investigating TFR; and more recently, Nicolini et al. [23], using a droplet dPCR platform for BCR-ABL1 monitoring, identified an IS threshold of $\geq 0.0023 \%$ to predict patients with a higher risk of molecular relapse. They also prospectively demonstrated that the DMR measured with dPCR is a predictive factor for the MR maintenance after TKI interruption [12, 16, 23]. We obviously are in favor of introducing dPCR into clinical practice, for instance, by promoting studies to evaluate comparatively qPCR and dPCR. The use of more sensitive diagnostic tools [24] could push the inclusion criteria of TKI stopping trials and may reduce the number of failures [25, 26].

It is extremely important to acquire information from long-term MRD monitoring, independently from the method. Gottschalk et al. [26] demonstrated that the individual molecular dynamics during TKI dose reduction is a promising predictor of molecular recurrence after TKI cessation. Spiess et al. [25] strongly suggest that by modifying and improving the methodology for detecting BCR-ABL1, even using RT-qPCR, the number of patients enrolled in stopping trials increases, due to a redefinition of the molecular response classes. Clark [27] has recently reported that gradual treatment tapering may improve TFR success, and that it may be possible to extend TFR attempts to patients who are in stable major molecular response but not necessarily $\mathrm{MR}^{4.0}$.

Our case report faces a dilemma and raises a question: which of the two methods reflects the patient's status of MRD? Our experience suggests that RT-qPCR, applied to MRD monitoring of patients undergoing TKI stopping clinical trials, may overestimate the rate of patients experiencing MR loss, by determining the early exit of a setting of them. On the other hand, dPCR suggests that the amount of BCR-ABL1 transcript is maintained stable or reduced over time probably due to the administration of therapy, even at a reduced dose. We have already reported how the duration of TKI therapy and DMR correlate using APCR [16] but not with RT-qPCR monitoring. The answer to the question cannot come from the observation of a single case but only from systematic comparative studies that can and should be done in the context of prospective clinical trials. 


\section{Acknowledgements}

Thanks to Dr. Fabio Stagno, University of Catania (Italy), for scientific support.

\section{Statement of Ethics}

The NP-DPCR-CML_1603 clinical trial obtained local Ethical Committee approval. The OPTkIMA trial (ClinicalTrials.gov: NCT02326311) obtained local Ethical Committee approval. Our patient gave her written informed consent for the enrollment into both clinical trials and for the publication of her case.

\section{Conflict of Interest Statement}

The authors declare that they have no competing interests.

\section{Funding Sources}

This project was not supported by dedicated funding sources.

\section{References}

1 Hehlmann R, Hochhaus A, Baccarani M; LeukemiaNet European. Chronic myeloid leukaemia. Lancet. 2007; 370(9584):342-50.

2 Kantarjian H, O’Brien S, Jabbour E, Garcia-Manero G, Quintas-Cardama A, Shan J, et al. Improved survival in chronic myeloid leukemia since the introduction of imatinib therapy: a single-institution historical experience. Blood. 2012;119(9):1981-7.

3 Baccarani M, Deininger MW, Rosti G, Hochhaus A, Soverini S, Apperley JF, et al. European LeukemiaNet recommendations for the management of chronic myeloid leukemia: 2013. Blood. 2013;122(6):872-84.

4 Höglund M, Sandin F, Hellstrom K, Björeman M, Björkhom M, Brune M, et al. Tyrosine kinase inhibitor usage, treatment outcome, and prognostic scores in CML: report from the population-based Swedish CML registry. Blood. 2013;122:1284-92.

5 Apperley JF. Chronic myeloid leukaemia. Lancet. 2015;385(9976):1447-59.

6 National Comprehensive Cancer Network: NCCN Clinical Practice Guidelines in Oncology: Chronic Myeloid Leukemia, version 1. 2015. Available from: https://www.nccn.org/patients/.

7 Sasaki K, Strom SS, O'Brien S, Jabbour E, Ravandi F, Konopleva M, et al. Relative survival in patients with chronic-phase chronic myeloid leukaemia in the tyrosine-kinase inhibitor era: analysis of patient data from six prospective clinical trials. Lancet Haematol. 2015;2(5):e186-93.

8 Cross NC, White HE, Colomer D, Ehrencrona H, Foroni L, Gottardi E, et al. Laboratory recommendations for scoring deep molecular responses following treatment for chronic myeloid leukemia. Leukemia. 2015;29(5): 999-1003.

9 Izzo B, Gottardi EM, Errichiello S, Daraio F, Baratè C, Galimberti S. Monitoring chronic myeloid leukemia: how molecular tools may drive therapeutic approaches. Front Oncol. 2019;9:833.

10 Efficace F, Baccarani M, Breccia M, Alimena G, Rosti G, Cottone F, et al. Health-related quality of life in chronic myeloid leukemia patients receiving long-term therapy with imatinib compared with the general population. Blood. 2011;118(17):4554-60.

11 Mori S, Vagge E, Le Coutre P, Abruzzese E, Martino B, Pungolino E, et al. Age and dPCR can predict relapse in CML patients who discontinued imatinib: the ISAV study. Am J Hematol. 2015;90(10):910-4.

12 Bernardi S, Ruggieri G, Malagola M, Cancelli V, Cattina F, Polverelli N, et al. Digital PCR (Dpcr) a step forward to detection and quantification of minimal residual disease (MRD) in Ph+/BCR-ABL1 chronic myeloid leukemia (CML). J Mol Biomark Diagn. 2017;08(03):1-3.

13 Huggett JF, Cowen S, Foy CA. Considerations for digital PCR as an accurate molecular diagnostic tool. Clin Chem. 2015;61(1):79-88.

14 Cao L, Cui X, Hu J, Li Z, Choi JR, Yang Q, et al. Advances in digital polymerase chain reaction (dPCR) and its emerging biomedical applications. Biosens Bioelectron. 2017;90:459-74. 


\section{Case Reports in Oncology}

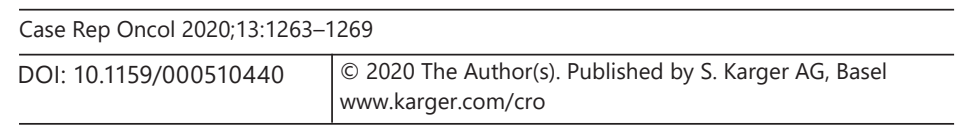

Zanaglio et al.: The Impact of $\mathrm{dPCR}$ on the Management of CML Patients

15 Alikian M, Whale AS, Akiki S, Piechocki K, Torrado C, Myint T, et al. RT-qPCR and RT-digital PCR: a comparison of different platforms for the evaluation of residual disease in chronic myeloid leukemia. Clin Chem. 2017; 63(2):525-31.

16 Bernardi S, Malagola M, Zanaglio C, Polverelli N, Dereli Eke E, D’Adda M, et al. Digital PCR improves the quantitation of DMR and the selection of CML candidates to TKIs discontinuation. Cancer Med. 2019;8(5):2041-55.

17 Bernardi S, Bonifacio M, Iurlo A, Zanaglio C, Tiribelli M, Binotto G, et al. Variant-specific discrepancy when quantitating BCR-ABL1 e13a2 and e14a2 transcripts using the Europe Against Cancer qPCR assay. Is dPCR the key? Eur J Haematol. 2019;103(3):272-3. https://doi.org/10.1111/ejh.13282.

18 Russo D, Martinelli G, Malagola M, Skert C, Soverini S, Iacobucci I, et al. Effects and outcome of a policy of intermittent imatinib treatment in elderly patients with chronic myeloid leukemia. Blood. 2013;121(26):5138-44.

19 Russo D, Malagola M, Skert C, Cancelli V, Turri D, Pregno P, et al. Managing chronic myeloid leukaemia in the elderly with intermittent imatinib treatment. Blood Cancer J. 2015;5(9):e347.

20 Branford S, Fletcher L, Cross NC, Müller MC, Hochhaus A, Kim DW, et al. Desirable performance characteristics for BCR-ABL measurement on an international reporting scale to allow consistent interpretation of individual patient response and comparison of response rates between clinical trials. Blood. 2008;112(8):3330.

21 Steegmann JL, Baccarani M, Breccia M, Casado LF, García-Gutiérrez V, Hochhaus A, et al. European LeukemiaNet recommendations for the management and avoidance of adverse events of treatment in chronic myeloid leukaemia. Leukemia. 2016;30(8):1648-71.

22 Gabert J, Beillard E, van der Velden VH, Bi W, Grimwade D, Pallisgaard N, et al. Standardization and quality control studies of "real-time" quantitative reverse transcriptase polymerase chain reaction of fusion gene transcripts for residual disease detection in leukemia - a Europe Against Cancer program. Leukemia. 2003; 17(12):2318-57.

23 Nicolini FE, Dulucq S, Boureau L, Cony-Makhoul P, Charbonnier A, Escoffre-Barbe M, et al. Evaluation of residual disease and TKI duration are critical predictive factors for molecular recurrence after stopping imatinib first-line in chronic phase CML patients. Clin Cancer Res. 2019;25(22):6606-13.

24 Bernardi Simona, Malagola Michele, Polverelli Nicola, Russo Domenico. Exosomes in Chronic Myeloid Leukemia: Are We Reading a New Reliable Message? Acta Haematol.. 1421-9662. 2020;143(5):509-510. 10.1159/000505088. 31922494.

25 Spiess B, Rinaldetti S, Naumann N, Galuschek N, Kossak-Roth U, Wuchter P, et al. Diagnostic performance of the molecular BCR-ABL1 monitoring system may impact on inclusion of CML patients in stopping trials. PLoS One. 2019;14(3):e0214305.

26 Gottschalk A, Glauche I, Cicconi S, Clark RE, Roeder I. Molecular monitoring during dose reduction predicts recurrence after TKI cessation in CML. Blood. 2020;135(10):766-9.

27 Clark RE. Tyrosine kinase inhibitor therapy discontinuation for patients with chronic myeloid leukaemia in clinical practice. Curr Hematol Malig Rep. 2019;14(6):507-14. 